\title{
Cosmic Redshift, Temperature, Growth Rate and Age in Stoney Scale Black Hole Cosmology
}

\author{
U. V. S. Seshavatharam ${ }^{1,2^{*}}$, S. Lakshiminarayana ${ }^{3}$ \\ ${ }^{1}$ Honorary Faculty, I-SERVE, Alakapuri, Hyderabad, India \\ ${ }^{2}$ QA, DIP Division, Lanco Industries Ltd, Rachagunneri, Srikalahasti, India \\ ${ }^{3}$ Department of Nuclear Physics, Andhra University, Visakhapatnam, India \\ Email: 'seshavatharam.uvs@gmail.com, Insrirama@yahoo.com
}

Received 5 June 2014; revised 10 July 2014; accepted 15 September 2014

Copyright (C) 2014 by authors and OALib.

This work is licensed under the Creative Commons Attribution International License (CC BY). http://creativecommons.org/licenses/by/4.0/

(c) (i) Open Access

\section{Abstract}

Throughout the cosmic evolution, currently believed cosmic "critical density" can be shown to be a default result of the "positively curved" light speed rotating black hole universe "mass density". As there is no observational or experimental evidence to Friedmann's second assumption, the density classification scheme of Friedmann cosmology must be reviewed at fundamental level and possibly can be relinquished. With cosmic light speed rotation galactic rotational curves can be understood. The observed cosmic redshift can be reinterpreted as an index of "cosmological" thermodynamic light emission mechanism. Clearly speaking during cosmic evolution, at any time in the past, in hydrogen atom-emitted photon energy was always inversely proportional to the cosmic temperature. Thus past light emitted from older galaxy's excited hydrogen atom will show redshift with reference to the current laboratory data. Note that there will be no change in the energy of the emitted photon during its journey from the distant galaxy to the observer. Past high cosmic temperature may be the root cause of observed super novae dimming. By considering the "Stoney mass" as the initial mass of the baby cosmic black hole, past and current physical and thermal parameters (like angular velocity, growth rate, age, redshift, thermal energy density and matter density) of the cosmic black hole can be understood. For a past cosmic temperature of $3000 \mathrm{~K}$, obtained redshift is 1100 . From now onwards, CMBR temperature can be called as "Comic Black Hole's Thermal Radiation" temperature and can be expressed as "CBHTR" temperature. Current cosmic black hole is growing at a rate of $14.66 \mathrm{~km} / \mathrm{sec}$ in a decelerating mode. In view of the confirmed zero rate of change, the current CMBR temperature (from satellite data) and zero rate of change in the current Hubble's constant (from satellite data), it can be suggested that, current cosmic expansion is almost all saturated and at present there is no significant cosmic acceleration. From microscopic physics point of view, $\left[\frac{\mathrm{d}(\hbar)}{\mathrm{d} t}\right.$ or $\left.\frac{\mathrm{d}}{\mathrm{d} t}\left(\frac{1}{\alpha}\right)\right]$ can be considered as a true in-

\footnotetext{
${ }^{*}$ Corresponding author.
} 


\section{dex of future cosmic rate of expansion.}

\section{Keywords}

Mach's Principle, Stoney Mass, Black Hole Cosmology, Cosmic Growth Index, Cosmic Growth Rate,
Hubble Potential, Cosmic Redshift, Cosmic Age, MOND \& Galactic Rotational Curves Subject Areas: Quantum Mechanics, Theoretical Physics

\section{Introduction}

The 3 great facts of cosmology were: galactic redshift, cosmic microwave background radiation temperature and super novae dimming. It may be noted that: 1 ) If it is true that galaxy constitutes so many stars, each star constitutes so many hydrogen atoms and light is coming from the excited electron of galactic hydrogen atom, then considering redshift as an index of "whole galaxy" receding may not be reasonable; 2) If one is willing to consider the CMBR temperature as a quantum gravitational result, then general theory of relativity and quantum mechanics can be unified into a scale independent quantum cosmology; 3) According to standard cosmology, since decoupling, the temperature of the CMBR temperature has dropped by a factor of roughly 1100 due to the expansion of the universe. As the universe expands, the CMB photons are redshifted, making the radiation's temperature inversely proportional to a parameter called the universe's scale factor. If $T_{t}$ is the temperature of the CMB and $z$ is the observed redshift, then $T_{t} \cong(1+z) 2.725 \mathrm{~K}$ where $(1+z)$ is known as the universal scale factor. Extending this concept, it can also be possible to guess that emitted quantum of energy is inversely proportional to the CMB temperature. If so, aged and distant super novae dimming effect can also be considered as a result of high CBR temperature. Based on these 3 points, the subject of standard cosmology can be reviewed at fundamental level.

So far no ground based laboratory has confirmed the individual existence of "dark matter" and "dark energy". Till today "quantum cosmology" has been in its beginning stage. One of the difficulties of quantum gravity is that quantum gravitational effects are only expected to become apparent near the Planck scale. Current status of quantum gravity seems to be highly theoretical and experimental verification is beyond the scope of current technology. If theoretical predictions are not in line with the observations, then either observations have to be interpreted in a different manner or theory has to be modified as per the observations. In this context, quantum gravity can be considered as a key tool. Clearly speaking by considering the subject of "black hole cosmology" as a key branch of the quantum gravity, many fundamental issues of theoretical and observational cosmology can be understood. In this context authors published their concepts on black hole cosmology in many online journals [1].

In this paper by reviewing the basics of Friedmann cosmology [2] an attempt is made to understand the model of black hole cosmology [3] [4] in terms of cosmic redshift [5] [6], CMBR redshift, cosmic growth index, cosmic growth rate and cosmic age. The paper is organized in the following order. Section 1 deals with the basic shortcomings of standard Friedmann cosmology. Section 2 deals with possible assumptions and the key features of black hole cosmology. Section 3 deals with the cosmic thermal energy density, matter density and the age along with a detailed table for the estimated cosmological physical, thermal and redshift data beginning from the Stoney scale. Section 4 deals with the galactic rotational curves. Finally Section 5 deals with the proposed conclusions.

The basic shortcomings of modern cosmology can be expressed as follows. For more information one may please refer [1].

1) There is no direct observational evidence to Friedmann's second assumption and no theoretical base in Friedmann's "critical density" concept and the "matter density" classification scheme. Clearly speaking, when Friedmann's cosmology was taking its final shape, black hole physics was in its beginning stage.

2) If light is coming from the atoms of the gigantic galaxy, then redshift can also be interpreted as an index of the galactic cosmological atomic "light emission mechanism". In no way does it seem to be connected with "galaxy receding". Instead of "wavelength" based redshift definition, in terms of "quantum of energy", redshift can also be interpreted as an index of cosmological temperature dependent light emission mechanism in hydrogen atom. 
3) Merely by estimating galaxy distance and without measuring galaxy receding speed, one cannot verify its receding speed or acceleration. (Clearly speaking: two mistakes are possible here. 1) Assumed galaxy receding speed is not being measured and not being confirmed; 2) Without measuring and confirming the galaxy receding speed, how can one say and confirm that it (galaxy) is accelerating.) There is no direct observational evidence for the current cosmic acceleration and the dark energy.

4) No theoretical base in considering the Hubble's constant merely as the cosmic expansion parameter. With reference to angular velocity it is having deep inner meaning. Note that, modern cosmologists believe that the rate of the change of the Hubble constant describes how fast/slow the Hubble constant changes over time and this rate does not tell if the Universe is currently expanding. This logic seems to be misleading. In authors opinion, if magnitude of past Hubble's constant was higher than the current magnitude then magnitude of past $\left(c / H_{t}\right)$ will be smaller than the current Hubble length $\left(c / H_{0}\right)$. If so rate of the decrease of the Hubble constant can be considered as a true index of rate of increase in Hubble length and thus with reference to Hubble length, rate of the decrease of the Hubble constant can be considered as a true index of cosmic rate of expansion. Proceeding further -in future, certainly with reference to current Hubble's constant, $\mathrm{d}\left(c / H_{0}\right) / \mathrm{d} t$ gives the true cosmic rate of expansion. Same logic can be applied to cosmic back ground temperature also. Clearly speaking $\mathrm{d}\left(T_{0}\right) / \mathrm{d} t$ gives the true cosmic rate of expansion. To understand the ground reality, sensitivity and accuracy of current methods of estimating the magnitudes of $\left(H_{0}\right.$ and $T_{0}$ ) must be improved.

5) By substituting the geometric mean mass of $\left(c^{3} / 2 G H_{0}\right)$ and $\sqrt{h c / 2 \pi G}$ in the famous Hawking's black hole temperature formula automatically the observed $2.725 \mathrm{~K}$ can be fitted very accurately.

6) No comparative and relational study in between Friedmann cosmology and microscopic physical phenomena

Friedmann made two simple assumptions about the universe. They can be stated in the following way.

1) When viewed at large enough scales, universe appears the same in every direction.

2) When viewed at large enough scales, universe appears the same from every location.

In this regard Hawking says [7]: "There is no scientific evidence for the Friedmann's second assumption. We believe it only on grounds of modesty: it would be most remarkable if the universe looked the same in every direction around us, but not around other points in the universe." This is one key point to be noted here. The term "critical density" is the back bone of modern cosmology. At any time in the past, it is generally expressed in the following way.

$$
\left(\rho_{c}\right)_{t} \cong \frac{3 H_{t}^{2}}{8 \pi G}
$$

Its current expression is as follows.

$$
\left(\rho_{c}\right)_{0} \cong \frac{3 H_{0}^{2}}{8 \pi G}
$$

According to standard Friedmann cosmology,

1) If matter density is greater than the critical density, universe will have a positive curvature.

2) If matter density equals the critical density, universe will be flat.

3) If matter density is less than the critical density, universe will have a negative curvature.

But by considering "black hole geometry" as the "eternal cosmic geometry" and by assuming "constant light speed rotation" throughout the cosmic evolution, at any time the currently believed cosmic "critical density" can be shown to be the cosmic black hole's eternal "mass density". If mass of the black hole universe is $M_{t},\left(\frac{c}{H_{t}}\right)$ is the radius of the black hole universe that rotates at light speed and angular velocity $H_{t}$, at any time in the past,

$$
\begin{gathered}
\frac{2 G M_{t}}{c^{2}} \cong \frac{c}{H_{t}} \text { and } M_{t} \cong \frac{c^{3}}{2 G H_{t}} \\
\left(\rho_{v}\right)_{t} \cong\left(M_{t}\right)\left[\frac{4 \pi}{3}\left(\frac{c}{H_{t}}\right)^{3}\right]^{-1} \cong\left(\frac{c^{3}}{2 G H_{t}}\right)\left[\frac{3}{4 \pi}\left(\frac{H_{t}}{c}\right)^{3}\right] \cong \frac{3 H_{t}^{2}}{8 \pi G}
\end{gathered}
$$

At present, 


$$
\left(\rho_{v}\right)_{0} \cong\left(M_{0}\right)\left[\frac{4 \pi}{3}\left(\frac{c}{H_{0}}\right)^{3}\right]^{-1} \cong\left(\frac{c^{3}}{2 G H_{0}}\right)\left[\frac{3}{4 \pi}\left(\frac{H_{0}}{c}\right)^{3}\right] \cong \frac{3 H_{0}^{2}}{8 \pi G}
$$

Based on this coincidence and as there is no observational or experimental evidence to Friedmann's second assumption, the density classification scheme of Friedmann cosmology must be reviewed at fundamental level.

\section{Possible Assumptions}

Possible assumptions in unified cosmic black hole physics can be expressed in the following way.

Assumption-1: With reference to the elementary charge and with mass similar to the Planck mass, a new mass unit can be constructed in the following way. It can be called as the Stoney mass [8].

$$
\left(M_{S}\right)^{ \pm} \cong \sqrt{\frac{\mathrm{e}^{2}}{4 \pi \varepsilon_{0} G}} \cong 1.859272 \times 10^{-9} \mathrm{Kg} \cong 1.042975 \times 10^{18} \mathrm{GeV} / \mathrm{c}^{2}
$$

Assumption-2: At any time Hubble length $\left(c / H_{t}\right)$ can be considered as the gravitational or electromagnetic interaction range.

Assumption-3: At any time, $H_{t}$ being the angular velocity, universe can be considered as a growing and light speed rotating primordial black hole. Thus at any given cosmic time,

$$
R_{t} \cong \frac{2 G M_{t}}{c^{2}} \cong \frac{c}{H_{t}} \quad \text { and } \quad M_{t} \cong \frac{c^{3}}{2 G H_{t}}
$$

when

$$
M_{t} \rightarrow M_{S}, \quad R_{S} \cong \frac{2 G M_{S}}{c^{2}} \quad \text { and } \quad H_{S} \cong \frac{c}{R_{S}} \cong \frac{c^{3}}{2 G M_{S}}
$$

can be considered as the characteristic initial physical measurements of the universe. Here the subscript $S$ refers to the initial conditions of the universe and can be called as the Stony scale. Similarly

$$
R_{0} \cong \frac{2 G M_{0}}{c^{2}} \cong \frac{c}{H_{0}}, \quad M_{0} \cong \frac{c^{3}}{2 G H_{0}} \quad \text { and } \quad H_{0} \cong \frac{c^{3}}{2 G M_{0}}
$$

can be considered as the characteristic current physical measurements of the universe.

Assumption-4: During cosmic evolution, at any time the past, in hydrogen atom emitted photon energy was always inversely proportional to the cosmic temperature. Thus past light emitted from older galaxy's hydrogen atom will show redshift with reference to the current laboratory data. There will be no change in the energy of the emitted photon during its journey from the distant galaxy to the observer.

$$
\left.\begin{array}{l}
\frac{E_{0}}{E_{t}} \cong \frac{\lambda_{t}}{\lambda_{0}} \cong \frac{T_{t}}{T_{0}} \cong\left(z_{0}+1\right) \\
z_{0} \cong \frac{E_{0}-E_{t}}{E_{t}} \cong \frac{\lambda_{t}-\lambda_{0}}{\lambda_{0}} \cong \frac{T_{t}-T_{0}}{T_{0}}
\end{array}\right\}
$$

Here, $E_{t}$ is the energy of emitted photon from the galactic hydrogen atom and $E_{0}$ is the corresponding energy in the laboratory. $\lambda_{t}$ is the wave length of emitted and received photon from the galactic hydrogen atom and $\lambda_{0}$ is the corresponding wave length in the laboratory. $T_{t}$ is the cosmic temperature at the time when the photon was emitted and is $T_{0}$ the current cosmic temperature and $z_{0}$ is the current redshift.

Assumption-5: At any given time, ratio of volume energy density and thermal energy density can be called as the cosmic growth index and can be expressed as follows.

$$
\frac{3 H_{t}^{2} c^{2}}{8 \pi G a T_{t}^{4}} \cong\left[1+\ln \left(\frac{M_{t}}{M_{S}}\right)\right]^{2} \cong\left[1+\ln \left(\frac{H_{S}}{H_{t}}\right)\right]^{2} \cong \text { Cosmic Growth index }
$$


Thus at the Stoney scale,

$$
\frac{3 H_{S}^{2} c^{2}}{8 \pi G a T_{S}^{4}} \cong\left[1+\ln \left(\frac{M_{S}}{M_{S}}\right)\right]^{2} \cong\left[1+\ln \left(\frac{H_{S}}{H_{S}}\right)\right]^{2} \cong 1
$$

Assumption-6: At any given time, cosmic black hole's growth rate can be expressed as $g_{t} \cong\left(\frac{3 H_{t}^{2} c^{2}}{8 \pi G a T_{t}^{4}}\right)^{-1}$ c.

With this idea and by considering the average growth rate cosmic age can be estimated.

$$
\begin{aligned}
g_{t} & \cong \text { Cosmic growth rate } \cong \frac{c}{\text { cosmic growth index }} \cong\left(\frac{3 H_{t}^{2} c^{2}}{8 \pi G a T_{t}^{4}}\right)^{-1} c \\
& \cong\left[1+\ln \left(\frac{M_{t}}{M_{S}}\right)\right]^{-2} \quad c \cong\left[1+\ln \left(\frac{H_{S}}{H_{t}}\right)\right]^{-2} c
\end{aligned}
$$

At the Stoney scale,

$$
g_{S} \cong\left(\frac{3 H_{S}^{2} c^{2}}{8 \pi G a T_{S}^{4}}\right)^{-1} c \cong\left[1+\ln \left(\frac{M_{S}}{M_{S}}\right)\right]^{-2} c \cong\left[1+\ln \left(\frac{H_{S}}{H_{S}}\right)\right]^{-2} c \cong c
$$

\subsection{To Reinterpret the Hubble's Constant}

With a simple derivation it is possible to show that, Hubble's constant $H_{t}$ represents the cosmological angular velocity. Authors presented this derivation in their published papers. Basic idea of this derivation is to express the angular velocity of any rotating celestial body in terms of its mass, radius, mass density and surface escape velocity. Assume that, a planet of mass $M$ and radius $R$ rotates with angular velocity $\omega_{e}$ and linear velocity $v_{e}$ in such a way that, free or loosely bound particle of mass $m$ lying on its equator gains a kinetic energy equal to potential energy as,

$$
\begin{gathered}
\frac{1}{2} m v_{e}^{2}=\frac{G M m}{R} \\
R \omega_{e}=v_{e}=\sqrt{\frac{2 G M}{R}} \text { and } \omega_{e}=\frac{v_{e}}{R}=\sqrt{\frac{2 G M}{R^{3}}}
\end{gathered}
$$

i.e. Linear velocity of planet's rotation is equal to free particle's escape velocity. Without any external power or energy, test particle gains escape velocity by virtue of planet's rotation. Note that if Earth completes one rotation in one hour then free particles lying on the equator will get escape velocity. Now writing $M=\frac{4 \pi}{3} R^{3} \rho_{e}$,

$$
\begin{gathered}
\omega_{e}=\frac{v_{e}}{R}=\sqrt{\frac{8 \pi G \rho_{e}}{3}} \text { or } \omega_{e}^{2}=\frac{8 \pi G \rho_{e}}{3} \\
\text { Density, } \rho_{e}=\frac{3 \omega_{e}^{2}}{8 \pi G}
\end{gathered}
$$

In real time, this obtained density may or may not be equal to the actual density. But the ratio $\frac{8 \pi G \rho_{\text {real }}}{3 \omega_{\text {real }}^{2}}$ may have some physical significance. The most important point to be noted here, is that, as far as dimensions and units are considered, from Equation (18), it is very clear that, proportionality constant being $\frac{3}{8 \pi G}$,

$$
\text { density } \propto(\text { angular velocity })^{2}
$$


Equation (18) is similar to "flat model concept" of cosmic "critical density"

$$
\rho_{c}=\frac{3 H_{t}^{2}}{8 \pi G}
$$

Comparing Equations (18) and (20) dimensionally and conceptually, i.e.

$$
\begin{gathered}
\rho_{e}=\frac{3 \omega_{e}^{2}}{8 \pi G} \quad \text { with } \quad \rho_{c}=\frac{3 H_{t}^{2}}{8 \pi G} \\
H_{t}^{2} \rightarrow \omega_{e}^{2} \quad \text { and } \quad H_{t} \rightarrow \omega_{e}
\end{gathered}
$$

It is very clear that, dimensions of "Hubble’s constant” must be "radian/second”. In any physical system under study, for any one "simple physical parameter" there will not be two different units and there will not be two different physical meanings. This is a simple clue and brings "cosmic rotation" into picture. This is possible in a closed universe only. Cosmic models that depend on this "critical density" may consider "angular velocity of the universe" in the place of "Hubble's constant". In the sense, with a great confidence "cosmic rotation" can be included in the existing models of cosmology. Then the term "critical density" appears to be the "volume density" of the closed and expanding universe. Thinking in this way, considering "black hole geometry" as the "eternal cosmic geometry" and by assuming "constant light speed rotation" throughout the cosmic evolution, at any time the currently believed cosmic "critical density" can be shown to be the cosmic black hole's eternal "volume density"'. Thus based on the Mach's principle, "distance cosmic back ground" can be quantified in terms of "Hubble volume" and "Hubble mass".

\subsection{To Reinterpret the Cosmic Redshift}

Hubble initially interpreted red shifts [5] as a Doppler effect, due to the motion of the galaxies as they receded for our location in the Universe. He called it a "Doppler effect" as though the galaxies were moving "through space"; that is how some astronomers initially perceived it. This is different to what has now become accepted but observations alone could not distinguish between the two concepts. In 1947 he [7] stated that: "The red shifts are more easily interpreted as evidence of motion in the line of sight away from the earth—as evidence that the nebulae in all directions are rushing away from us and that the farther away they are, the faster they are receding. This interpretation lends itself directly to theories of expanding universe. The interpretation is not universally accepted, but even the most cautious of us admit that red shifts are evidence of either an expanding universe or of some hitherto unknown principle of nature". "Attempts have been made to attain the necessary precision with the 100 inch, and the results appear to be significant. If they are valid, it seems likely that the red-shifts may not be due to an expanding universe, and much of the current speculation on the structure of the universe may require re-examination. The significant data, however, were necessarily obtained at the very limit of a single instrument, and there were no possible means of checking the results by independent evidence. Therefore the results must be accepted for the present as suggestive rather than definitive". "We may predict with confidence that the 200 inch will tell us whether the red shifts must be accepted as evidence of a rapidly expanding universe, or attributed to some new principle in nature. Whatever may be the answer, the result may be welcomed as another major contribution to the exploration of the universe."

In this regard if one is willing to consider the proposed assumptions, in hydrogen atom emitted photon energy can be understood as follows.

1) As the cosmic time increases cosmic angular velocity and hence cosmic temperature both decrease. As a result, during cosmic evolution, in hydrogen atom, binding energy increases in between proton and electron.

2) As cosmic temperature decreases, it requires more excitation energy to break the bond between electron and the proton. In this way, during cosmic evolution, whenever it is excited, hydrogen atom emits photons with increased quantum of energy. Past high cosmic temperature may be the root cause of the observed super novae dimming.

3) Thus past light quanta emitted from old galaxy's excited hydrogen atom will have less energy and show a red shift with reference to the current laboratory magnitude.

4) During journey light quanta will not lose energy and there will be no change in light wavelength.

5) Galactic photon energy in hydrogen atom when it was emitted can be estimated as follows. 


$$
E_{t} \cong \frac{h c}{\lambda_{t}} \cong\left(\frac{T_{0}}{T_{t}}\right)\left(\frac{h c}{\lambda_{0}}\right) \cong\left(\frac{T_{0}}{T_{t}}\right) E_{0}
$$

Here, $\lambda_{0}$ is the wavelength of photon in the laboratory.

$E_{t}$ is the energy of received photon when it was emitted in the distant galaxy.

$E_{0}$ is the corresponding energy of photon in the current laboratory methods.

$\lambda_{t}$ is the wavelength of emitted and received photon when it was emitted in the distant galaxy.

$T_{t}$ is the cosmic temperature at the time when the photon was emitted and is $T_{0}$ the current cosmic temperature.

From laboratory point of view, above concept can be understood in the following way. After some time in future,

$$
z_{f} \cong \frac{E_{f}-E_{0}}{E_{0}} \cong \frac{E_{f}}{E_{0}}-1
$$

Here, $E_{f}$ is the energy of photon emitted from laboratory hydrogen atom after some time in future. $E_{0}$ is the energy of current photon emitted from laboratory hydrogen atom. $z_{f}$ is the redshift of laboratory hydrogen atom after some time in future. In future-within the scope of experimental accuracy of laboratory hydrogen atom's redshift- $\left[\mathrm{d}\left(\mathrm{z}_{f}\right) / \mathrm{d} t\right]$ can be considered as a true index of absolute rate of cosmic expansion. It can be understood from Table 1 in the following way.

In Subsection 2.4 an attempt is made to understand the cosmological thermodynamic light emission mechanism in hydrogen atom in a unified approach.

\subsection{To Reinterpret the Hubble's Law}

Based on the assumptions it is possible to say that, during cosmic evolution, at any time, any galaxy will have revolution speed as well as receding speed simultaneously and both can be expressed in the following way.

$$
\left(V_{g}\right)_{\text {revolution }} \cong\left(\frac{r_{g}}{R_{t}}\right) c \cong r H_{t} \text { where } \quad r_{g} \leq\left(R_{t} \cong \frac{c}{H_{t}}\right)
$$

$r_{g}$ is the distance between galaxy and the cosmic center and $R_{t}$ is the cosmic radius at time $t$.

$$
\left(v_{g}\right)_{\text {receding }} \cong\left(\frac{r_{g}}{R_{t}}\right) g_{t} \cong\left(\frac{r_{g}}{R_{t}}\right)\left[1+\ln \left(\frac{H_{S}}{H_{t}}\right)\right]^{-2} c \cong\left[1+\ln \left(\frac{H_{S}}{H_{t}}\right)\right]^{-2} r_{g} H_{t} \cong\left[1+\ln \left(\frac{H_{S}}{H_{t}}\right)\right]^{-2}\left(V_{g}\right)_{\text {revolution }}
$$

Thus at present it can be suggested that, $\frac{\left(V_{g}\right)_{\text {revolution }}}{\left(v_{g}\right)_{\text {receding }}} \cong\left[1+\ln \left(\frac{H_{S}}{H_{t}}\right)\right]^{2}$. Please note that above two relations are independent of the observed redshift. This is for further study.

\subsection{To Understand the Cosmological Thermodynamic Light Emission Mechanism}

It is very tempting to make an analogy between the status of the cosmological "Standard Model” and that

Table 1. To understand the true nature of cosmic expansion.

Nature of change

Increasing

$$
\frac{\mathrm{d}\left(z_{f}\right)}{\mathrm{d} t}
$$

\section{Constant}

Decreasing
Nature of cosmic expansion

Acceleration

Uniform rate

Deceleration 
of particle physics [9]. In cosmology there are about 10 free parameters, each of which is becoming well determined, and with a great deal of consistency between different measurements. However, none of these parameters can be calculated from a fundamental theory, and so hints of the bigger picture, "physics beyond the Standard Model", are being searched for with ever more ambitious experiments. Despite this analogy, there are some basic differences. For one thing, many of the cosmological parameters change with cosmic epoch, and so the measured values are simply the ones determined today, and hence they are not "constants", like particle masses for example (although they are deterministic, so that if one knows their values at one epoch, they can be calculated at another). Moreover, the parameter set is not as well defined as it is in the particle physics Standard Model; different researchers will not necessarily agree on which parameters should be considered as free, and the set can be extended as the quality of the data improves. In a more general sense, the cosmological "Standard Model" is much further from the underlying "fundamental theory", which will ultimately provide the values of the parameters from first principles. Nevertheless, any genuinely complete "theory of everything" must include an explanation for the values of these cosmological parameters as well as the parameters of the Standard Model of particle physics. Current magnitude of Hubble constant [10] is $(67.80 \pm 0.77) \mathrm{km} / \mathrm{sec} / \mathrm{Mpc},(68.1 \pm 1.2)$ $\mathrm{km} / \mathrm{sec} / \mathrm{Mpc},(67.3 \pm 1.2) \mathrm{km} / \mathrm{sec} / \mathrm{Mpc},(69.7 \pm 2.0) \mathrm{km} / \mathrm{sec} / \mathrm{Mpc},(70.0 \pm 2.2) \mathrm{km} / \mathrm{sec} / \mathrm{Mpc},(70.6 \pm 3.3)$ $\mathrm{km} / \mathrm{sec} / \mathrm{Mpc},(73.8 \pm 2.4) \mathrm{km} / \mathrm{sec} / \mathrm{Mpc}$, and $(72.5 \pm 2.5) \mathrm{km} / \mathrm{sec} / \mathrm{Mpc}$. In a cosmological approach with various trial-error methods, at present in hydrogen atom, if $H_{0} \cong 71 \mathrm{~km} / \mathrm{sec} / \mathrm{Mpc}$, Bohr radius can be fitted as follows.

$$
\begin{aligned}
\left(a_{B}\right)_{0} & \cong\left(\frac{4 \pi \varepsilon_{0} G m_{p}^{2}}{\mathrm{e}^{2}}\right)\left(\frac{G M_{0}}{c^{2}}\right) \cong\left(\frac{4 \pi \varepsilon_{0} G m_{p}^{2}}{\mathrm{e}^{2}}\right)\left(\frac{c}{2 H_{0}}\right) \cong\left(\frac{4 \pi \varepsilon_{0} G m_{p}^{2}}{\mathrm{e}^{2}}\right)\left(\frac{c}{2 H_{0}}\right) \cong \frac{1}{2}\left(\frac{4 \pi \varepsilon_{0} G m_{p}^{2}}{\mathrm{e}^{2}}\right)\left(\frac{c}{H_{0}}\right) \\
& \cong 5.27225 \times 10^{-11} \mathrm{~m} .
\end{aligned}
$$

$\left(\frac{\mathrm{e}^{2}}{4 \pi \varepsilon_{0} G m_{p}^{2}}\right)$ is the electromagnetic and gravitational force ratio of proton. This relation seems to be very simple and needs no further derivation. Factor 2 may be connected with half of the current Hubble length $\left(\frac{1}{2} \frac{c}{H_{0}}\right)$. For any physicist or cosmologist it will be a very big surprise. Note that, this relation is free from the famous reduced Planck's constant, electron rest mass and other arbitrary numbers or coefficients. After simplification and considering the ground state, it is possible to express the ground state potential energy of electron in the following way.

$$
\left(E_{\mathrm{pot}}\right)_{0} \cong-\left(\frac{\mathrm{e}^{2}}{4 \pi \varepsilon_{0} G m_{p}^{2}}\right)\left(\frac{\mathrm{e}^{2} c^{2}}{4 \pi \varepsilon_{0} G M_{0}}\right) \cong-\left(\frac{\mathrm{e}^{2}}{4 \pi \varepsilon_{0} G m_{p}^{2}}\right)\left(\frac{\mathrm{e}^{2}}{4 \pi \varepsilon_{0}}\right)\left(\frac{1}{2} \frac{c}{H_{0}}\right)^{-1} \cong-2\left(\frac{\mathrm{e}^{2}}{4 \pi \varepsilon_{0} G m_{p}^{2}}\right)\left(\frac{\mathrm{e}^{2} H_{0}}{4 \pi \varepsilon_{0} c}\right)
$$

Here $\left(\frac{\mathrm{e}^{2} H_{0}}{4 \pi \varepsilon_{0} c}\right)$ can be called as the current Hubble potential. Characteristic ground state kinetic energy of electron can be expressed in the following way.

$$
\left(E_{\text {kin }}\right)_{0} \cong\left(\frac{\mathrm{e}^{2}}{4 \pi \varepsilon_{0} G m_{p}^{2}}\right)\left(\frac{\mathrm{e}^{2} c^{2}}{8 \pi \varepsilon_{0} G M_{0}}\right) \cong\left(\frac{\mathrm{e}^{2}}{4 \pi \varepsilon_{0} G m_{p}^{2}}\right)\left(\frac{\mathrm{e}^{2}}{4 \pi \varepsilon_{0}}\right)\left(\frac{c^{2}}{2 G M_{0}}\right) \cong\left(\frac{\mathrm{e}^{2}}{4 \pi \varepsilon_{0} G m_{p}^{2}}\right)\left(\frac{\mathrm{e}^{2} H_{0}}{4 \pi \varepsilon_{0} c}\right)
$$

Characteristic ground state total energy of electron can be expressed in the following way.

$$
\left(E_{\text {tot }}\right)_{0} \cong-\left(\frac{\mathrm{e}^{2}}{4 \pi \varepsilon_{0} G m_{p}^{2}}\right)\left(\frac{\mathrm{e}^{2} c^{2}}{8 \pi \varepsilon_{0} G M_{0}}\right) \cong-\left(\frac{\mathrm{e}^{2}}{4 \pi \varepsilon_{0} G m_{p}^{2}}\right)\left(\frac{\mathrm{e}^{2}}{4 \pi \varepsilon_{0}}\right)\left(\frac{c^{2}}{2 G M_{0}}\right) \cong-\left(\frac{\mathrm{e}^{2}}{4 \pi \varepsilon_{0} G m_{p}^{2}}\right)\left(\frac{\mathrm{e}^{2} H_{0}}{4 \pi \varepsilon_{0} c}\right)
$$

If $H_{0} \cong 71 \mathrm{~km} / \mathrm{sec} / \mathrm{Mpc},\left(E_{\text {tot }}\right)_{0} \cong-13.66 \mathrm{eV}$. Based on this coincidence, this proposed new concept can be given some consideration and it can be suggested that the best value of $H_{0}$ lies in between 70 and $71 \mathrm{~km} / \mathrm{sec} / \mathrm{Mpc}$. Unfortunately these relations seem to be independent of the reduced Planck's constant. If one is willing to linkup these relations with the observed "discrete" energy spectrum of the hydrogen atom, then the desired cosmological 
light emission mechanism can be developed in a unified picture. Considering the concept of stationary orbits and jumping nature of electron, emitted photon energy can be expressed in the following way.

$$
\left(E_{\text {photon }}\right)_{0} \cong\left(\frac{\mathrm{e}^{2}}{4 \pi \varepsilon_{0} G m_{p}^{2}}\right)\left(\frac{\mathrm{e}^{2} H_{0}}{4 \pi \varepsilon_{0} c}\right)\left[\frac{1}{n_{1}^{2}}-\frac{1}{n_{2}^{2}}\right]
$$

where $n_{1}=n_{2} \cong 1,2,3, \cdots$ and $n_{2}>n_{1}$. The best fit of $H_{0}$ can be obtained in the following way.

$$
\left.\begin{array}{l}
\left(\frac{\mathrm{e}^{2}}{4 \pi \varepsilon_{0} G m_{p}^{2}}\right)\left(\frac{\mathrm{e}^{2} H_{0}}{4 \pi \varepsilon_{0} c}\right) \cong \frac{\mathrm{e}^{4} m_{e}}{32 \pi^{2} \varepsilon_{0}^{2} \hbar^{2}} \\
\text { and } H_{0} \cong \frac{G m_{p}^{2} m_{e} c}{2 \hbar^{2}} \cong 70.738 \mathrm{~km} / \mathrm{sec} / \mathrm{Mpc}
\end{array}\right\}
$$

At any time in the past—in support of the proposed cosmological red shift interpretation, above relations can be re-expressed as follows.

$$
\begin{gathered}
\left(E_{\mathrm{pot}}\right)_{t} \cong-\left(\frac{T_{0}}{T_{t}}\right)\left(\frac{\mathrm{e}^{2}}{4 \pi \varepsilon_{0} G m_{p}^{2}}\right)\left(\frac{\mathrm{e}^{2} c^{2}}{4 \pi \varepsilon_{0} G M_{0}}\right) \cong-2\left(\frac{T_{0}}{T_{t}}\right)\left(\frac{\mathrm{e}^{2}}{4 \pi \varepsilon_{0} G m_{p}^{2}}\right)\left(\frac{\mathrm{e}^{2} H_{0}}{4 \pi \varepsilon_{0} c}\right) \\
\left(E_{\text {kin }}\right)_{t} \cong\left(\frac{T_{0}}{T_{t}}\right)\left(\frac{\mathrm{e}^{2}}{4 \pi \varepsilon_{0} G m_{p}^{2}}\right)\left(\frac{\mathrm{e}^{2} H_{0}}{4 \pi \varepsilon_{0} c}\right) \\
\left(E_{\text {tot }}\right)_{t} \cong-\left(\frac{T_{0}}{T_{t}}\right)\left(\frac{\mathrm{e}^{2}}{4 \pi \varepsilon_{0} G m_{p}^{2}}\right)\left(\frac{\mathrm{e}^{2} H_{0}}{4 \pi \varepsilon_{0} c}\right)
\end{gathered}
$$

This can be considered as the base for the "cosmological thermodynamic light emission mechanism”. At any time in the past, at any galaxy, emitted photon energy can be expressed as follows.

$$
\left(E_{\text {photon }}\right)_{t} \cong\left(\frac{T_{0}}{T_{t}}\right)\left(\frac{\mathrm{e}^{2}}{4 \pi \varepsilon_{0} G m_{p}^{2}}\right)\left(\frac{\mathrm{e}^{2} H_{0}}{4 \pi \varepsilon_{0} c}\right)\left[\frac{1}{n_{1}^{2}}-\frac{1}{n_{2}^{2}}\right]_{t}
$$

This issue is for further study. In a unified picture, with reference to the current cosmic temperature, electron's current quantum of angular momentum can be expressed as follows.

$$
\hbar \cong m_{p} \sqrt{\frac{G m_{e} c}{2 H_{0}}} \cong \frac{G m_{p} \sqrt{m_{e} M_{0}}}{c} \cong \hbar_{0}
$$

From microscopic physics point of view, $\left[\frac{\mathrm{d}(\hbar)}{\mathrm{d} t}\right.$ or $\left.\frac{\mathrm{d}}{\mathrm{d} t}\left(\frac{1}{\alpha}\right)\right]$ can be considered as a true index of future cosmic rate of expansion. If atomic nuclear mass increases in integral multiples of the proton mass, then the observed discreteness of the reduced Planck’s constant can be expressed as follows.

$$
n \hbar \cong \frac{G\left(n \cdot m_{p}\right) \sqrt{m_{e} M_{0}}}{c} \cong n \hbar_{0}
$$

where $n=1,2,3, \cdots$ This issue is also for further study. At any time in the past, hypothetically, in terms of the current and past "primordial" cosmic temperatures, it is possible to express the cosmological "variable quantum of angular momentum" of electron in the following way. Whether it is virtual or real or speculative- to be confirmed from further study.

$$
\hbar_{t} \cong \sqrt{\frac{T_{t}}{T_{0}}} \cdot \hbar_{0} \cong \sqrt{\frac{\lambda_{t}}{\lambda_{0}}} \cdot \hbar_{0}
$$


It may be noted that, throughout the cosmic evolution, Planck's constant and the Uncertainty constant both can be conside-red as "constants". Now the fundamental questions to be answered are:

1) Is reduced Planck's constant—an output of the atomic system?

2) Is the reduced Planck's constant-a cosmological variable?

3) Is the Planck's constant-a cosmological constant?

4) How to understand and how to consider the constancy of the Planck's constant along with the variable reduced Planck's constant?

5) Is the condition, $\hbar \rightarrow(h / 2 \pi)$ an indication of saturation or halt of cosmological expansion?

\section{Connecting Cosmic Thermal and Physical Parameters}

\subsection{Cosmic Thermal Energy Density and Matter Energy Density}

It may be noted that connecting CMBR energy density with Hubble's constant is really a very big task and mostly preferred in cosmology. At any given cosmic time, thermal energy density can be expressed with the following semi empirical relation.

$$
\begin{gathered}
a T_{t}^{4} \cong\left[1+\ln \left(\frac{M_{t}}{M_{S}}\right)\right]^{-2}\left(\frac{3 H_{t}^{2} c^{2}}{8 \pi G}\right) \cong\left[1+\ln \left(\frac{H_{S}}{H_{t}}\right)\right]^{-2}\left(\frac{3 H_{t}^{2} c^{2}}{8 \pi G}\right) \\
T_{t} \cong\left[1+\ln \left(\frac{H_{S}}{H_{t}}\right)\right]^{-\frac{1}{2}}\left(\frac{3 H_{t}^{2} c^{2}}{8 \pi G a}\right)^{\frac{1}{4}}
\end{gathered}
$$

With a suitable derivation if above expression is obtained, then certainly the subject of black hole cosmology is put into main stream physics. Thus at present, if $H_{0}$ is close to $71 \mathrm{~km} / \mathrm{sec} / \mathrm{Mpc}$, obtained CMBR temperature is $2.723 \mathrm{~K}$. For the time being this can be considered as a remarkable discovery and an accurate fit.

$$
\begin{gathered}
a T_{0}^{4} \cong\left[1+\ln \left(\frac{H_{S}}{H_{t}}\right)\right]^{-2}\left(\frac{3 H_{0}^{2} c^{2}}{8 \pi G}\right) \cong\left[1+\ln \left(\frac{M_{0}}{M_{S}}\right)\right]^{-2}\left(\frac{3 H_{0}^{2} c^{2}}{8 \pi G}\right) \\
T_{0} \cong\left[1+\ln \left(\frac{H_{S}}{H_{0}}\right)\right]^{-\frac{1}{2}}\left(\frac{3 H_{0}^{2} c^{2}}{8 \pi G a}\right)^{\frac{1}{4}}
\end{gathered}
$$

With this coincidence it can be suggested that, at the beginning of cosmic evolution,

$$
a T_{S}^{4} \cong\left(\frac{3 H_{S}^{2} c^{2}}{8 \pi G}\right)
$$

With reference to the current cosmic temperature, at any time in the past,

$$
\frac{T_{t}}{T_{0}} \cong\left\{\frac{\left[1+\ln \left(\frac{H_{S}}{H_{0}}\right)\right] H_{t}}{\left[1+\ln \left(\frac{H_{S}}{H_{t}}\right)\right] H_{0}}\right\}^{\frac{1}{2}}
$$

Using this relation, cosmic redshift data can be fitted. When the assumed CMBR temperature is $2999 \mathrm{~K}$, estimated redshift is 1099 and is in very good agreement with the standard model of cosmology.

Thus at any time based on $\left[\frac{\mathrm{d}}{\mathrm{d} t}\left(T_{t}\right)\right.$ and $\left.\frac{\mathrm{d}}{\mathrm{d} t}\left(H_{t}\right)\right]$, the absolute cosmic rate of expansion can be confirmed. At present with reference to $\left[\frac{\mathrm{d}}{\mathrm{d} t}\left(T_{0}\right)\right.$ and $\left.\frac{\mathrm{d}}{\mathrm{d} t}\left(H_{0}\right)\right]$ current "true" cosmic rate of expansion can be understood. 
Drop in current "cosmic temperature" can be considered as a measure of the current cosmic expansion and "rate of decrease in current cosmic temperature" can be considered as a measure of the current cosmic "rate of expansion". But if rate of decrease in temperature is very small and is beyond the scope of current experimental verification, then the two possible states are: a) cosmic temperature is decreasing at a very slow rate and universe is expanding at a very slow rate and b) there is no "observable" thermal expansion and there is no "observable" cosmic expansion. If observed CMBR temperature is $2.725 \mathrm{~K}$ and is very low in magnitude and is very close to absolute zero, then thinking about and confirming the "cosmic acceleration" may not be reasonable. Similarly rate of decrease in current "Hubble's constant" can be considered as a measure of current cosmic "rate of expansion". If rate of decrease in current "Hubble's constant" is very small and is beyond the scope of current experimental verification, then the two possible states are: a) current "Hubble's constant" is decreasing at a very slow rate and current universe is expanding at a very slow rate and b) at present there is no "observable" cosmic expansion. Fortunately as per the Cobe/Planck satellite data current CMBR temperature is very smooth and isotropic, and there is no data that refers to the rate of change in the current Hubble's constant. Hence it can be suggested that at present there is no significant cosmic expansion. Even though this suggestion is completely against to the current notion of cosmic acceleration [11], based on the proposed arguments, relations and observed data authors request the science community to review the standard cosmology. If observed CMB radiation temperature is $2.725 \mathrm{~K}$ and is very low in magnitude and is very close to absolute zero, then thinking about and confirming the "cosmic acceleration" may not be reasonable. Mostly at the ending stage of expansion, rate of change in $H_{t}$ will be practically zero and can be considered as practically constant. Thus at its ending stage of expansion, for the whole cosmic black hole as $H_{t}$ practically remains constant, its corresponding thermal energy density will be "the same" throughout its volume. This "sameness" may be the reason for the observed "isotropic" nature of the current $\mathrm{CMB}$ radiation.

Matter-energy density can be considered as the geometric mean density of volume energy density and the thermal energy density and it can be expressed with the following semi empirical relation.

$$
\left(\rho_{m}\right)_{t} c^{2} \cong \sqrt{\left(\frac{3 H_{t}^{2} c^{2}}{8 \pi G}\right)\left(a T_{t}^{4}\right)} \cong\left[1+\ln \left(\frac{H_{S}}{H_{t}}\right)\right]^{-1}\left(\frac{3 H_{t}^{2} c^{2}}{8 \pi G}\right) \cong\left[1+\ln \left(\frac{M_{t}}{M_{S}}\right)\right]^{-1}\left(\frac{3 H_{0}^{2} c^{2}}{8 \pi G}\right)
$$

Here one important observation to be noted is that, at any time

$$
\frac{8 \pi G\left(\rho_{m}\right)_{t}}{3 H_{t}^{2}} \cong\left(\Omega_{m}\right)_{t} \cong\left[1+\ln \left(\frac{M_{t}}{M_{S}}\right)\right]^{-1} \cong\left[1+\ln \left(\frac{H_{S}}{H_{t}}\right)\right]^{-1}
$$

Thus at present,

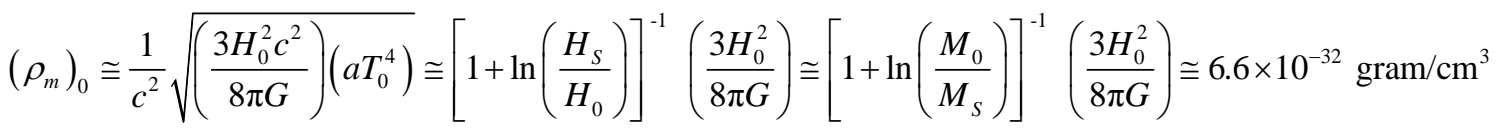

Based on the average mass-to-light ratio for any galaxy present matter density can be expressed with the following relation [12].

$$
\left(\rho_{m}\right)_{0} \cong 1.5 \times 10^{-32} \eta h_{0} \mathrm{gram} / \mathrm{cm}^{3}
$$

Here $\eta \cong\left\langle\frac{M}{L}\right\rangle_{\text {galaxy }} /\left\langle\frac{M}{L}\right\rangle_{\text {sun }}, h_{0} \cong H_{0} / 100 \mathrm{Km} / \mathrm{sec} / \mathrm{Mpc} \cong 0.71$ Note that elliptical galaxies probably comprise about $60 \%$ of the galaxies in the universe and spiral galaxies thought to make up about $20 \%$ percent of the galaxies in the universe. Almost $80 \%$ of the galaxies are in the form of elliptical and spiral galaxies. For spiral galaxies, $\eta h_{0}^{-1} \cong 9 \pm 1$ and for elliptical galaxies, $\eta h_{0}^{-1} \cong 10 \pm 2$ For our galaxy inner part, $\eta h_{0}^{-1} \cong 6 \pm 2$. Thus the average $\eta h_{0}^{-1}$ is very close to 8 to 9 and its corresponding matter density is close to $(6.0$ to 6.7$) \times 10^{-32}$ $\mathrm{gram} / \mathrm{cm}^{3}$ and can be compared with the above proposed magnitude of $6.6 \times 10^{-32} \mathrm{gram} / \mathrm{cm}^{3}$.

\subsection{Age of the Growing Cosmic Black Hole}

Age of the growing cosmic black hole can be assumed as the time taken to grow from the assumed Stoney scale to 
the current scale. At present,

$$
g_{0} \cong\left(\frac{8 \pi G a T_{0}^{4}}{3 H_{0}^{2} c^{2}}\right) c \cong\left[1+\ln \left(\frac{M_{0}}{M_{S}}\right)\right]^{-2} c \cong\left[1+\ln \left(\frac{H_{S}}{H_{0}}\right)\right]^{-2} \quad c \simeq 14.66 \mathrm{~km} / \mathrm{sec}
$$

Clearly speaking, at present, Hubble volume is growing at $14.66 \mathrm{~km} / \mathrm{sec}$ in a decelerating trend. Starting from the Stoney scale, if the assumed growth rate is gradually decreasing, at any time average growth rate can be expressed as follows.

$$
\frac{g_{S}+g_{t}}{2} \cong \frac{1}{2}\left\{1+\left[1+\ln \left(\frac{M_{t}}{M_{S}}\right)\right]^{-2}\right\} c \cong \frac{1}{2}\left\{1+\left[1+\ln \left(\frac{H_{S}}{H_{t}}\right)\right]^{-2}\right\} c
$$

For the current scale, average growth rate can be expressed as follows.

$$
\frac{g_{S}+g_{0}}{2} \cong \frac{1}{2}\left\{1+\left[1+\ln \left(\frac{M_{0}}{M_{S}}\right)\right]^{-2}\right\} c \cong \frac{1}{2}\left\{1+\left[1+\ln \left(\frac{H_{S}}{H_{0}}\right)\right]^{-2}\right\} c
$$

Time taken to reach from the Stoney scale to any assumed scale can be expressed as follows.

$$
\left(\frac{g_{S}+g_{t}}{2}\right) t \cong\left(R_{t}-R_{S}\right) \cong R_{t}
$$

where, $R_{t} \gg R_{S}$ and $R_{S} \approx 0$ Hence for the current scale,

$$
\begin{gathered}
\left(\frac{g_{S}+g_{0}}{2}\right) t_{0} \cong\left(R_{0}-R_{S}\right) \cong R_{0} \cong \frac{c}{H_{0}} \\
t_{0} \cong\left(\frac{g_{S}+g_{0}}{2}\right)^{-1} \frac{c}{H_{0}} \cong\left\{1+\left[1+\ln \left(\frac{H_{S}}{H_{0}}\right)\right]^{-2}\right\}^{-1} \frac{2}{H_{0}} \cong 27.496 \mathrm{Gyr}
\end{gathered}
$$

where $\left\{1+\left[1+\ln \left(\frac{H_{S}}{H_{0}}\right)\right]^{-2}\right\}^{-1} \cong 0.99995$. This proposal is for further study. Based on this proposal, after one second from the Stoney scale, cosmic angular velocity is $2 \mathrm{rad} / \mathrm{sec}$, growth rate is $29 \mathrm{~km} / \mathrm{sec}$ and cosmic temperature is $3 \times 10^{9} \mathrm{~K}$.

With reference to the current and past cosmic temperatures, at any time in the past, at any galaxy, for any hydrogen atom,

$$
\frac{E_{0}}{E_{t}} \cong \frac{\lambda_{t}}{\lambda_{0}} \cong \frac{T_{t}}{T_{0}} \cong\left\{\frac{\left[1+\ln \left(\frac{H_{S}}{H_{0}}\right)\right] H_{t}}{\left[1+\ln \left(\frac{H_{S}}{H_{t}}\right)\right] H_{0}}\right\}^{\frac{1}{2}} \cong\left\{\frac{\left(\Omega_{m}\right)_{t} H_{t}}{\left(\Omega_{m}\right)_{0} H_{0}}\right\}^{\frac{1}{2}}
$$

By guessing $H_{t},\left(z_{0}+1\right)$ can be estimated. It seems to be a full and absolute definition for the cosmic redshift. Thus at any time in the past,

$$
\left(\frac{E_{0}}{E_{t}}-1\right) \cong\left(\frac{\lambda_{t}}{\lambda_{0}}-1\right) \cong\left(\frac{T_{t}}{T_{0}}-1\right) \cong\left\{\frac{\left[1+\ln \left(\frac{H_{S}}{H_{0}}\right)\right] H_{t}}{\left[1+\ln \left(\frac{H_{S}}{H_{t}}\right)\right] H_{0}}\right\}^{\frac{1}{2}}-1 \cong\left\{\frac{\left(\Omega_{m}\right)_{t} H_{t}}{\left(\Omega_{m}\right)_{0} H_{0}}\right\}^{\frac{1}{2}}-1 \cong z_{0}
$$


One can see Table 2 for the estimated cosmological physical, thermal and redshift data beginning form the Stoney scale. As a guide, this data can be compared with the standard cosmology data for further study and analysis.

This table prepared with $\mathrm{C}++$ program with reference to the observed $2.725 \mathrm{~K}$. In this table:

Column-1 = Assumed cosmic angular velocity. Column-2 = Estimated cosmic radius, from Relation (7).

Column-3 = Estimated cosmic mass, from Relation (7). Column-4 = Estimated cosmic growth index, from relation (10).

Column-5 = Estimated cosmic growth rate, from Relation (12). Column-6 = Estimated cosmic time, from relation (53).

Column-7 = Estimated cosmic temperature, from Relation (41). Column-8 = Estimated cosmic redshift, from relation (57)

Table 2. Assumed Cosmic angular velocity and estimated other cosmic physical and thermal parameters.

\begin{tabular}{|c|c|c|c|c|c|c|c|}
\hline $\begin{array}{l}\text { Assumed Cosmic } \\
\text { Angular velocity } \\
\text { (rad/sec) }\end{array}$ & $\begin{array}{l}\text { Estimated } \\
\text { Cosmic radius } \\
\text { (meter) }\end{array}$ & $\begin{array}{l}\text { Estimated } \\
\text { Cosmic } \\
\text { mass (kg) }\end{array}$ & $\begin{array}{l}\text { Cosmic Growth } \\
\text { index } \cong\left[1+\ln \left(\frac{H_{s}}{H_{t}}\right)\right]^{2} \\
\text { (number) }\end{array}$ & $\begin{array}{l}\text { Estimated } \\
\text { Cosmic Growth } \\
\text { rate }(\mathrm{km} / \mathrm{sec})\end{array}$ & $\begin{array}{l}\text { Estimated } \\
\text { Cosmic time } \\
\text { (sec) }\end{array}$ & $\begin{array}{l}\text { Estimated } \\
\text { Cosmic } \\
\text { temperature } \\
(\mathrm{K})\end{array}$ & $\begin{array}{c}\text { Estimated } \\
\text { Cosmic } \\
\text { Redshift } z_{0} \\
\text { (number) }\end{array}$ \\
\hline $1.086 \mathrm{E}+44$ & $2.761 \mathrm{E}-36$ & $1.859 \mathrm{E}-09$ & 1 & 299792 & $0.000 \mathrm{E}+00$ & $2.237 E+32$ & $8.207 E+31$ \\
\hline $2.305 E+43$ & $1.301 \mathrm{E}-35$ & $8.759 \mathrm{E}-09$ & 6.50173 & 46109.6 & $5.924 \mathrm{E}-44$ & $6.455 \mathrm{E}+31$ & $2.368 \mathrm{E}+31$ \\
\hline $2.305 E+42$ & $1.301 \mathrm{E}-34$ & $8.759 \mathrm{E}-08$ & 23.5461 & 12732.1 & $8.148 \mathrm{E}-43$ & $1.480 \mathrm{E}+31$ & $5.428 \mathrm{E}+30$ \\
\hline $2.305 E+41$ & $1.301 \mathrm{E}-33$ & $8.759 \mathrm{E}-07$ & 51.1943 & 5855.97 & $8.493 E-42$ & $3.853 \mathrm{E}+30$ & $1.414 \mathrm{E}+30$ \\
\hline $2.305 E+40$ & $1.301 \mathrm{E}-32$ & $8.759 \mathrm{E}-06$ & 89.4463 & 3351.65 & $8.580 \mathrm{E}-41$ & $1.060 \mathrm{E}+30$ & $3.888 E+29$ \\
\hline $2.305 E+39$ & $1.301 \mathrm{E}-31$ & $8.759 \mathrm{E}-05$ & 138.302 & 2167.66 & $8.615 \mathrm{E}-40$ & $3.006 \mathrm{E}+29$ & $1.103 E+29$ \\
\hline $2.305 E+38$ & $1.301 \mathrm{E}-30$ & $8.759 \mathrm{E}-04$ & 197.762 & 1515.93 & $8.634 \mathrm{E}-39$ & $8.692 E+28$ & $3.189 E+28$ \\
\hline $2.305 E+37$ & $1.301 \mathrm{E}-29$ & $8.759 \mathrm{E}-03$ & 267.825 & 1119.36 & $8.645 \mathrm{E}-38$ & $2.548 \mathrm{E}+28$ & $9.347 \mathrm{E}+27$ \\
\hline $2.305 E+36$ & $1.301 \mathrm{E}-28$ & $8.759 \mathrm{E}-02$ & 348.492 & 860.256 & $8.653 \mathrm{E}-37$ & $7.544 \mathrm{E}+27$ & $2.768 E+27$ \\
\hline $2.305 E+35$ & $1.301 \mathrm{E}-27$ & $8.759 \mathrm{E}-01$ & 439.763 & 681.714 & $8.658 \mathrm{E}-36$ & $2.251 \mathrm{E}+27$ & $8.258 \mathrm{E}+26$ \\
\hline $2.305 E+34$ & $1.301 \mathrm{E}-26$ & $8.759 \mathrm{E}+00$ & 541.638 & 553.492 & $8.662 \mathrm{E}-35$ & $6.756 \mathrm{E}+26$ & $2.479 \mathrm{E}+26$ \\
\hline $2.305 E+33$ & $1.301 \mathrm{E}-25$ & $8.759 \mathrm{E}+01$ & 654.116 & 458.317 & $8.665 E-34$ & $2.038 \mathrm{E}+26$ & $7.477 \mathrm{E}+25$ \\
\hline $2.305 E+32$ & $1.301 \mathrm{E}-24$ & $8.759 \mathrm{E}+02$ & 777.199 & 385.735 & $8.667 \mathrm{E}-33$ & $6.173 \mathrm{E}+25$ & $2.265 E+25$ \\
\hline $2.305 E+31$ & $1.301 \mathrm{E}-23$ & $8.759 E+03$ & 910.885 & 329.122 & $8.668 \mathrm{E}-32$ & $1.876 \mathrm{E}+25$ & $6.883 E+24$ \\
\hline $2.305 E+30$ & $1.301 \mathrm{E}-22$ & $8.759 E+04$ & 1055.17 & 284.116 & $8.670 \mathrm{E}-31$ & $5.719 \mathrm{E}+24$ & $2.098 E+24$ \\
\hline $2.305 E+29$ & $1.301 \mathrm{E}-21$ & $8.759 \mathrm{E}+05$ & 1210.07 & 247.748 & $8.671 \mathrm{E}-30$ & $1.748 \mathrm{E}+24$ & $6.411 \mathrm{E}+23$ \\
\hline $2.305 E+28$ & $1.301 \mathrm{E}-20$ & $8.759 E+06$ & 1375.57 & 217.941 & $8.671 \mathrm{E}-29$ & $5.352 \mathrm{E}+23$ & $1.964 \mathrm{E}+23$ \\
\hline $2.305 E+27$ & $1.301 \mathrm{E}-19$ & $8.759 \mathrm{E}+07$ & 1551.67 & 193.207 & $8.672 \mathrm{E}-28$ & $1.642 \mathrm{E}+23$ & $6.025 \mathrm{E}+22$ \\
\hline $2.305 E+26$ & $1.301 \mathrm{E}-18$ & $8.759 \mathrm{E}+08$ & 1738.37 & 172.456 & $8.673 \mathrm{E}-27$ & $5.048 \mathrm{E}+22$ & $1.852 \mathrm{E}+22$ \\
\hline $2.305 E+25$ & $1.301 \mathrm{E}-17$ & $8.759 \mathrm{E}+09$ & 1935.68 & 154.877 & $8.673 \mathrm{E}-26$ & $1.554 \mathrm{E}+22$ & $5.701 \mathrm{E}+21$ \\
\hline $2.305 E+24$ & $1.301 \mathrm{E}-16$ & $8.759 \mathrm{E}+10$ & 2143.59 & 139.855 & $8.674 \mathrm{E}-25$ & $4.790 \mathrm{E}+21$ & $1.757 \mathrm{E}+21$ \\
\hline $2.305 E+23$ & $1.301 \mathrm{E}-15$ & $8.759 \mathrm{E}+11$ & 2362.11 & 126.917 & $8.674 \mathrm{E}-24$ & $1.478 \mathrm{E}+21$ & $5.424 \mathrm{E}+20$ \\
\hline $2.305 E+22$ & $1.301 \mathrm{E}-14$ & $8.759 \mathrm{E}+12$ & 2591.23 & 115.695 & $8.674 \mathrm{E}-23$ & $4.568 \mathrm{E}+20$ & $1.676 \mathrm{E}+20$ \\
\hline $2.305 E+21$ & $1.301 \mathrm{E}-13$ & $8.759 E+13$ & 2830.96 & 105.898 & $8.675 \mathrm{E}-22$ & $1.413 \mathrm{E}+20$ & $5.184 \mathrm{E}+19$ \\
\hline $2.305 E+20$ & $1.301 \mathrm{E}-12$ & $8.759 E+14$ & 3081.28 & 97.2947 & $8.675 E-21$ & $4.375 E+19$ & $1.605 E+19$ \\
\hline
\end{tabular}




\section{Continued}

\begin{tabular}{|c|c|c|c|c|c|c|c|}
\hline $2.305 E+19$ & $1.301 \mathrm{E}-11$ & $8.759 E+15$ & 3342.21 & 89.6987 & $8.675 E-20$ & $1.356 \mathrm{E}+19$ & $4.973 \mathrm{E}+18$ \\
\hline $2.305 E+18$ & $1.301 \mathrm{E}-10$ & $8.759 \mathrm{E}+16$ & 3613.75 & 82.9588 & 8.675E-19 & $4.204 \mathrm{E}+18$ & $1.542 \mathrm{E}+18$ \\
\hline $2.305 E+17$ & $1.301 \mathrm{E}-09$ & 8.759E+17 & 3895.89 & 76.951 & $8.676 \mathrm{E}-18$ & $1.305 E+18$ & $4.786 \mathrm{E}+17$ \\
\hline $2.305 E+16$ & $1.301 \mathrm{E}-08$ & $8.759 \mathrm{E}+18$ & 4188.63 & 71.5729 & $8.676 \mathrm{E}-17$ & $4.052 \mathrm{E}+17$ & $1.486 \mathrm{E}+17$ \\
\hline $2.305 E+15$ & $1.301 \mathrm{E}-07$ & $8.759 \mathrm{E}+19$ & 4491.98 & 66.7395 & $8.676 \mathrm{E}-16$ & $1.259 \mathrm{E}+17$ & $4.619 \mathrm{E}+16$ \\
\hline $2.305 E+14$ & $1.301 \mathrm{E}-06$ & $8.759 E+20$ & 4805.93 & 62.3797 & $8.676 \mathrm{E}-15$ & $3.915 E+16$ & $1.436 \mathrm{E}+16$ \\
\hline $2.305 E+13$ & $1.301 \mathrm{E}-05$ & $8.759 \mathrm{E}+21$ & 5130.48 & 58.4336 & $8.676 \mathrm{E}-14$ & $1.218 \mathrm{E}+16$ & $4.468 \mathrm{E}+15$ \\
\hline $2.305 E+12$ & $1.301 \mathrm{E}-04$ & $8.759 \mathrm{E}+22$ & 5465.64 & 54.8504 & $8.676 \mathrm{E}-13$ & $3.791 \mathrm{E}+15$ & $1.391 \mathrm{E}+15$ \\
\hline $2.305 \mathrm{E}+11$ & $1.301 \mathrm{E}-03$ & $8.759 \mathrm{E}+23$ & 5811.41 & 51.5869 & 8.676E-12 & $1.180 \mathrm{E}+15$ & $4.331 \mathrm{E}+14$ \\
\hline $2.305 \mathrm{E}+10$ & $1.301 \mathrm{E}-02$ & $8.759 E+24$ & 6167.77 & 48.6063 & 8.676E-11 & $3.678 \mathrm{E}+14$ & $1.349 \mathrm{E}+14$ \\
\hline $2.305 E+09$ & $1.301 \mathrm{E}-01$ & $8.759 \mathrm{E}+25$ & 6534.74 & 45.8767 & $8.676 \mathrm{E}-10$ & $1.146 \mathrm{E}+14$ & $4.206 \mathrm{E}+13$ \\
\hline $2.305 E+08$ & $1.301 \mathrm{E}+00$ & $8.759 \mathrm{E}+26$ & 6912.31 & 43.3708 & 8.677E-09 & $3.575 E+13$ & $1.311 \mathrm{E}+13$ \\
\hline $2.305 \mathrm{E}+07$ & $1.301 \mathrm{E}+01$ & $8.759 \mathrm{E}+27$ & 7300.49 & 41.0647 & 8.677E-08 & $1.115 \mathrm{E}+13$ & $4.091 \mathrm{E}+12$ \\
\hline $2.305 \mathrm{E}+06$ & $1.301 \mathrm{E}+02$ & $8.759 E+28$ & 7699.27 & 38.9378 & $8.677 \mathrm{E}-07$ & $3.480 \mathrm{E}+12$ & $1.277 \mathrm{E}+12$ \\
\hline $2.305 E+05$ & $1.301 \mathrm{E}+03$ & $8.759 E+29$ & 8108.66 & 36.9719 & 8.677E-06 & $1.086 \mathrm{E}+12$ & $3.985 E+11$ \\
\hline $2.305 \mathrm{E}+04$ & $1.301 \mathrm{E}+04$ & $8.759 \mathrm{E}+30$ & 8528.65 & 35.1512 & 8.677E-05 & $3.392 \mathrm{E}+11$ & $1.244 \mathrm{E}+11$ \\
\hline $2.305 E+03$ & $1.301 \mathrm{E}+05$ & 8.759E+31 & 8959.24 & 33.4618 & $8.677 \mathrm{E}-04$ & $1.059 \mathrm{E}+11$ & $3.887 \mathrm{E}+10$ \\
\hline $2.305 E+02$ & $1.301 \mathrm{E}+06$ & $8.759 E+32$ & 9400.43 & 31.8913 & $8.677 \mathrm{E}-03$ & $3.310 \mathrm{E}+10$ & $1.214 \mathrm{E}+10$ \\
\hline $2.305 \mathrm{E}+01$ & $1.301 \mathrm{E}+07$ & $8.759 E+33$ & 9852.23 & 30.4289 & 8.677E-02 & $1.035 \mathrm{E}+10$ & $3.796 \mathrm{E}+09$ \\
\hline $2.305 \mathrm{E}+00$ & $1.301 \mathrm{E}+08$ & $8.759 \mathrm{E}+34$ & 10314.6 & 29.0648 & 8.677E-01 & $3.234 \mathrm{E}+09$ & $1.187 \mathrm{E}+09$ \\
\hline $2.305 \mathrm{E}-01$ & $1.301 \mathrm{E}+09$ & $8.759 \mathrm{E}+35$ & 10787.6 & 27.7904 & 8.677E+00 & $1.011 \mathrm{E}+09$ & $3.710 \mathrm{E}+08$ \\
\hline $2.305 \mathrm{E}-02$ & $1.301 \mathrm{E}+10$ & $8.759 E+36$ & 11271.3 & 26.598 & $8.677 \mathrm{E}+01$ & $3.163 E+08$ & $1.161 \mathrm{E}+08$ \\
\hline $2.305 \mathrm{E}-03$ & $1.301 \mathrm{E}+11$ & $8.759 \mathrm{E}+37$ & 11765.5 & 25.4807 & 8.677E+02 & $9.897 \mathrm{E}+07$ & $3.631 \mathrm{E}+07$ \\
\hline $2.305 E-04$ & $1.301 \mathrm{E}+12$ & $8.759 E+38$ & 12270.3 & 24.4324 & $8.677 \mathrm{E}+03$ & $3.097 \mathrm{E}+07$ & $1.136 \mathrm{E}+07$ \\
\hline $2.305 E-05$ & $1.301 \mathrm{E}+13$ & $8.759 \mathrm{E}+39$ & 12785.7 & 23.4475 & $8.677 \mathrm{E}+04$ & $9.693 \mathrm{E}+06$ & $3.556 \mathrm{E}+06$ \\
\hline $2.305 E-06$ & $1.301 \mathrm{E}+14$ & $8.759 \mathrm{E}+40$ & 13311.7 & 22.5209 & $8.677 \mathrm{E}+05$ & $3.034 \mathrm{E}+06$ & $1.113 \mathrm{E}+06$ \\
\hline $2.305 E-07$ & $1.301 \mathrm{E}+15$ & $8.759 E+41$ & 13848.4 & 21.6482 & $8.677 \mathrm{E}+06$ & $9.501 \mathrm{E}+05$ & $3.486 \mathrm{E}+05$ \\
\hline $2.305 \mathrm{E}-08$ & $1.301 \mathrm{E}+16$ & $8.759 \mathrm{E}+42$ & 14395.6 & 20.8253 & $8.677 \mathrm{E}+07$ & $2.976 \mathrm{E}+05$ & $1.092 \mathrm{E}+05$ \\
\hline $2.305 \mathrm{E}-09$ & $1.301 \mathrm{E}+17$ & $8.759 E+43$ & 14953.4 & 20.0484 & $8.677 \mathrm{E}+08$ & $9.321 \mathrm{E}+04$ & $3.419 \mathrm{E}+04$ \\
\hline $2.305 \mathrm{E}-10$ & $1.301 \mathrm{E}+18$ & $8.759 \mathrm{E}+44$ & 15521.9 & 19.3142 & 8.677E+09 & $2.920 \mathrm{E}+04$ & $1.071 \mathrm{E}+04$ \\
\hline 2.305E-11 & $1.301 \mathrm{E}+19$ & $8.759 \mathrm{E}+45$ & 16100.9 & 18.6196 & $8.677 \mathrm{E}+10$ & $9.150 \mathrm{E}+03$ & $3.356 \mathrm{E}+03$ \\
\hline $2.52 \mathrm{E}-12$ & 1.19E+20 & $8.01 \mathrm{E}+46$ & 16667.6 & 17.9865 & $7.94 \mathrm{E}+11$ & 2998.85 & 1099.21 \\
\hline $2.305 \mathrm{E}-12$ & $1.301 \mathrm{E}+20$ & $8.759 \mathrm{E}+46$ & 16690.6 & 17.9618 & 8.677E+11 & $2.868 \mathrm{E}+03$ & $1.051 \mathrm{E}+03$ \\
\hline $2.305 \mathrm{E}-13$ & $1.301 \mathrm{E}+21$ & $8.759 E+47$ & 17290.8 & 17.3382 & 8.677E+12 & $8.988 \mathrm{E}+02$ & $3.288 \mathrm{E}+02$ \\
\hline $2.305 \mathrm{E}-14$ & $1.301 \mathrm{E}+22$ & $8.759 E+48$ & 17901.7 & 16.7466 & 8.677E+13 & $2.818 \mathrm{E}+02$ & $1.024 \mathrm{E}+02$ \\
\hline $2.305 E-15$ & $1.301 \mathrm{E}+23$ & $8.759 E+49$ & 18523.2 & 16.1847 & 8.677E+14 & $8.835 E+01$ & $3.141 \mathrm{E}+01$ \\
\hline $2.305 E-16$ & $1.301 \mathrm{E}+24$ & $8.759 E+50$ & 19155.2 & 15.6507 & 8.677E+15 & $2.771 \mathrm{E}+01$ & $9.164 \mathrm{E}+00$ \\
\hline $2.305 \mathrm{E}-17$ & $1.301 \mathrm{E}+25$ & $8.759 \mathrm{E}+51$ & 19797.9 & 15.1427 & 8.677E+16 & $8.689 \mathrm{E}+00$ & $2.188 \mathrm{E}+00$ \\
\hline $2.305 E-18$ & $1.301 \mathrm{E}+26$ & $8.759 E+52$ & 20451.2 & 14.6589 & $8.677 \mathrm{E}+17$ & $2.726 \mathrm{E}+00$ & $0.000 \mathrm{E}+00$ \\
\hline
\end{tabular}




\section{Galactic Rotational Curves in the Current Black Hole Universe}

With reference to the results of the Modified Newtonian Dynamics [13] [14], empirically rotational speed of a star is being represented as

$$
v_{s} \cong \sqrt[4]{G M a_{0}}
$$

where $a_{0} \cong(1.2 \pm 0.3) \times 10^{-10} \mathrm{~m} \cdot \mathrm{sec}^{-2} \approx \mathrm{CH}_{0} / 2 \pi$, and $M$ is the mass of galaxy. In the light speed rotating black hole universe,

1) The acceleration constant $a_{0}$ is not a constant but a variable and depends on the galactic revolving speed about the center of the light speed rotating black hole universe.

2) Its magnitude can be assumed to be proportional to the current hubble constant and can be called as the cosmological galactic acceleration.

3) By considering the galactic revolving speed $V_{g}$ about the center of the cosmic black hole, magnitude of $\left(\mathrm{cH}_{0}\right)$ can be assumed to vary in the following way.

$$
\left(V_{g} / c\right)\left(c H_{0}\right) \cong\left(V_{g} H_{0}\right) \cong a_{g} .
$$

Thus authors replace the empirical acceleration constant $a_{0}$ with (a variable) cosmological galactic acceleration, $a_{g} \cong V_{g} H_{0}$. Now rotational speed of a star in any galaxy can be represented as follows.

$$
v \cong \sqrt[4]{G M\left(V_{g} H_{0}\right)} \cong \sqrt[4]{G M r_{g} H_{0}^{2}}
$$

where $r_{g}$ is the current distance between the galaxy and the cosmic black hole center. Here if it is assumed that, galaxies under observation possesses a cosmological revolving speed in the range 0.1 to 0.25 times the speed of light, currently observed all galactic rotational speeds can be fitted well. Advantage of this proposal is that, by knowing the galactic mass and rotational speeds of its stars, galactic revolving speed and hence distance between galaxy and the cosmic black hole center can be estimated. This is for further study. It is true that this proposal is 1) Qualitatively suitable for understanding the galactic rotation curves in the light of light speed cosmic rotation; 2) By knowing the galactic rotational speeds quantitatively suitable for estimating the galactic cosmological revolution speed and distance from the cosmic center.

\section{Conclusions}

Based on the above proposed logics, concepts and data fitting, it can be concluded in the following way:

1) The basic idea of unification is: a) To minimize the number of physical constants and to merge a group of different fundamental constants into one compound physical constant with appropriate unified interpretation and b)

To merge and minimize various branches of physics. In this regard, instead of the Planck mass, $M_{S} \cong \sqrt{\mathrm{e}^{2} / 4 \pi \varepsilon_{0} G}$ can be considered as the nature's given true unified mass unit.

2) If "black hole geometry" is more intrinsic compared to the black hole "mass" and "density" parameters, if universe constitutes so many galaxies and if each galaxy constitutes a central growing and fast spinning black hole then considering universe as a "growing and light speed rotating primordial black hole" may not be far away from reality. If universe is having no black hole geometry, any massive body (which is bound to the universe) may not show a black hole structure. That is-black hole structure or geometry may be a subset of the cosmic geometry. At this juncture considering or rejecting this proposal completely depends on the observed cosmic redshift. Based on the relations proposed in Sections 2 and 3 observed cosmic redshift can be considered as a result of cosmological light emission mechanism. Authors are working on the assumed Hubble volume and Hubble mass in different directions with different applications that connect micro physics and macro physics. Based on the proposed applications and short comings of the standard model of cosmology [1], concepts of black hole cosmology may be given at least $99 \%$ priority.

3) In view of the applications proposed in Sections (2) to (4), observed zero rate of change in the "current CMBR temperature” (from Cobe/Planck satellite data) and zero rate of change in the "current Hubble's constant” (from Cobe/Planck satellite data) it can be suggested that, current cosmic expansion is almost all saturated and at present there is no significant cosmic acceleration. Clearly speaking, Stoney scale cosmic black hole's growth 
rate is equal to the speed of light and current cosmic black hole is growing at $14.66 \mathrm{~km} / \mathrm{sec}$ in a decelerating trend. From microscopic physics point of view, $\left[\frac{\mathrm{d}(\hbar)}{\mathrm{d} t}\right.$ or $\left.\frac{\mathrm{d}}{\mathrm{d} t}\left(\frac{1}{\alpha}\right)\right]$ can be considered as a true index of future cosmic rate of expansion. It can be also be possible to suggest that currently believed "dark energy" is a pure, "mathematical concept" and there exists no physical base behind its confirmation.

4) Based on the proposed light speed rotating universe and the Modified Newtonian Dynamics, galactic rotational speeds can fit and thus it can be suggested that, existence of "Drark matter" is also a pure, "mathematical concept" and there exists no physical base behind its confirmation.

\section{Acknowledgements}

The first author is indebted to professor K. V. Krishna Murthy, Chairman, Institute of Scientific Research on Vedas (I-SERVE), Hyderabad, India and Shri K. V. R. S. Murthy, former scientist IICT (CSIR) Govt. of India, Director, Research and Development, I-SERVE, for their valuable guidance and great support in developing this subject. Both the authors are very much thankful to the anonymous referees for their valuable comments and kind suggestions in improving and bringing this subject into current main stream online physics research.

\section{References}

[1] Seshavatharam, U.V.S. and Lakshminarayana, S. (2014) Basics of Black Hole Cosmology—First Critical Scientific Review. Physical Science International Journal, 4, 842-879.

[2] Friedmann, A. (1999) On the Curvature of Space. General Relativity and Gravitation, 31, 1991-2000. http://dx.doi.org/10.1023/A:1026751225741

[3] Zhang, T.X. and Frederic, C. (2013) Acceleration of Black Hole Universe. Astrophysics and Space Science, 349, 567573.

[4] Poplawski, N.J. (2010) Radial motion into an Einstein-Rosen bridge. Physics Letters B, 687, 110-113. http://dx.doi.org/10.1016/j.physletb.2010.03.029

[5] Hubble, E.P. (1929) A Relation between Distance and Radial Velocity among Extra-Galactic Nebulae. Proceedings of the National Academy of Sciences of the United States of America, 15, 168-173.

[6] Hubble, E.P. (1947) The 200-Inch Telescope and Some Problems It May Solve. Publications of the Astronomical Society of the Pacific, 59, 153-167.

[7] Hawking, S.W. (1988) A Brief History of Time. Bantam Dell Publishing Group, New York.

[8] Stoney, G.J. (1881) On the Physical Units of Nature. Philosophical Magazine, 11, 381-391. http://dx.doi.org/10.1080/14786448108627031

[9] Beringer, J., et al. (2012) Particle Data Group. Physical Review D, 86, 010001. http://dx.doi.org/10.1103/PhysRevD.86.010001

[10] (2011) The Accelerating Universe. The Royal Swedish Academy of Sciences. 2011 Nobel Prize in Physics. www.nobelprize.org/nobel_prizes/physics/laureates/2011/advanced-physicsprize2011.pdf

[11] Spergel, D.N., et al. Planck Data Reconsidered. http://arxiv.org/pdf/1312.3313.pdf

[12] Narlikar, J.V. (2002) Introduction to Cosmology. Cambridge University Press, Cambridge.

[13] Brownstein, J.R. and Moffat, J.W. (2006) Galaxy Rotation Curves Without Non-Baryonic Dark Matter. The Astrophysical Journal, 636, 721-741.

[14] Chadwick, E.A., et al. (2013) A Gravitational Theoretical Development Supporting MOND. Physical Review D, 88, 024036. 